\title{
Three years of cross-section measurements of $(n, x n)$ threshold reactions at TSL Uppsala and NPI Řež
}

\author{
O. Svoboda ${ }^{1,2, a}$, J. Vrzalová1,2, A. Krása ${ }^{1}$, A. Kugler ${ }^{1}$, M. Majerle ${ }^{1}$, and V. Wagner ${ }^{1,2}$ \\ 1 Nuclear Physics Institute of the Academy of Sciences of the Czech Republic PRI, \\ 25068 Rež near Prague, Czech Republic \\ 2 Faculty of Nuclear Sciences and Physical Engineering, Czech Technical University, \\ Břehová 7, 11519 Prague, Czech Republic
}

\begin{abstract}
We use $(\mathrm{n}, \mathrm{xn})$ threshold reactions in various materials to measure high energy neutron flux from spallation reactions. Unfortunately, no experimental cross-section data exist for reactions with $\mathrm{x}$ higher than four and neutron energies over $\sim 40 \mathrm{MeV}$. With the support from EFNUDAT we performed seven successful $(n, x n)$ cross-section measurements in two campaigns exploiting the quasi-monoenergetic neutron source at The Svedberg Laboratory in Uppsala, Sweden. Neutron energies from the ${ }^{7} \operatorname{Li}(\mathrm{p}, \mathrm{n}){ }^{7} \mathrm{Be}$ based source were in the region 22 to $94 \mathrm{MeV}$. We carried out additional four irradiations with neutron energies from 17 up to $34 \mathrm{MeV}$ using the quasi-monoenergetic neutron source of the Nuclear Physics Institute in Řež. We studied Al, Au, Bi, I, In, and Ta materials. Although we used different neutron sources, gamma detectors and foil geometries, we obtained consistent results. We observed good agreement with the data in EXFOR, so we believe we have understood and solved all possible sources of uncertainties.
\end{abstract}

\section{Motivation}

We take part in the international project called Energy plus Transmutation, where a massive lead target with uranium blanket is irradiated with relativistic proton and deuteron beams [1]. Activation detectors of $\mathrm{Al}, \mathrm{Au}, \mathrm{Bi}, \mathrm{In}$, and $\mathrm{Ta}$ are used to measure high energy neutrons from spallation reactions. For evaluation, cross-sections of used threshold reactions in activation detectors are needed. Due to the lack of experimental cross-section data for such reactions in the energy region over $30 \mathrm{MeV}$, (n, xn) cross-section measurements on above mentioned materials were proposed.

\section{Evolution of our cross-section measurements}

In 2007, a proposal on (n,xn) cross-section measurements by the means of activation analysis and gamma spectra measurement was submitted by us to the EFNUDAT (European Facilities for Nuclear Data Measurements) [2]. For this type of measurements a (quasi)monoenergetic neutron source with high intensity and well-known spectrum is needed. The ${ }^{7} \mathrm{Li}(\mathrm{p}, \mathrm{n})^{7} \mathrm{Be}$ based neutron source at The Svedberg Laboratory (TSL), Uppsala, Sweden seemed to be the most suitable for us. After the EFNUDAT decision to support these measurements, a pilot/testing measurement on a very similar neutron source at Nuclear Physics Institute (NPI), Řež, Czech Republic, was performed in May 2008. The neutron beam energy was $21.88 \mathrm{MeV}$. In this experiment our cross-section measurement method was verified

\footnotetext{
a e-mail: svoboda@ujf.cas.cz
}

This is an Open Access article distributed under the terms of the Creative Commons Attribution-Noncommercial License 3.0, which permits unrestricted use, distribution, and reproduction in any noncommercial medium, provided the original work is properly cited. 
and spectroscopic corrections for the measurements in Uppsala were prepared. Uppsala measurements were than performed with neutron beam energies 22, 47, and $94 \mathrm{MeV}$ in June 2008.

To fill in the gaps in the data up to $40 \mathrm{MeV}$, next three irradiations at NPI with neutron energies 17.5, 30.375 and 35.875 MeV were performed in August 2008, April 2009 and May 2009. After their successful evaluation, new measurements at TSL were proposed to fill in the gaps between 47 and $94 \mathrm{MeV}$. With the support from EFNUDAT further irradiations at TSL with energies 62, 70, 80, and $92 \mathrm{MeV}$ were performed in February 2010. The evaluation of these measurements has not been completed yet. Their results will be published later together with the rest of the data from previous experiments.

\section{Experimental apparatus}

Cross-sections of $(\mathrm{n}, \mathrm{xn}),(\mathrm{n}, \mathrm{p})$ and $(\mathrm{n}, \alpha)$ reactions in $\mathrm{Al}, \mathrm{Au}, \mathrm{Bi}, \mathrm{In}$, and Ta foils and $\mathrm{KIO}_{3}$ tablet were studied. In some irradiations samples from $\mathrm{Co}, \mathrm{Cu}, \mathrm{Fe}, \mathrm{Mg}, \mathrm{Ni}, \mathrm{Y}$, and $\mathrm{Zn}$ were also tested.

Both two used quasi-monoenergetic neutron sources were based on ${ }^{7} \mathrm{Li}(\mathrm{p}, \mathrm{n})^{7} \mathrm{Be}$ reaction with approximately $50 \%$ of the neutrons in the peak $2-3 \mathrm{MeV}$ wide. More detail description of used neutron sources, same as their neutron spectra, are available in the proceedings from the ND2010 conference [3]. Details about the sample dimensions, HPGe measurements and spectroscopic corrections can be also found in the same place.

\section{Cross-section results}

Products of $(\mathrm{n}, \mathrm{xn})$ threshold reactions were observed up the "x" equal to 10 , that means for example ${ }^{209} \mathrm{Bi}(\mathrm{n}, 10 \mathrm{n}){ }^{200} \mathrm{Bi}$. The highest observed isotope in gold was ${ }^{188} \mathrm{Au},{ }^{108} \mathrm{In}$ in natural indium, ${ }^{119} \mathrm{I}$ in iodine and ${ }^{176} \mathrm{Ta}$ in tantalum.

The following figures 1-7 present our cross-section data compared with TALYS 1.0 calculations and data from the EXFOR database. Numerical values with their uncertainties were published in the proceeding from ND2010 conference [3]. Good agreement with the data from EXFOR was observed, so it can be assumed that also the rest of measured cross-sections is reliably determined.

Systematic analysis of the EXFOR data is planned to distinguish between different experiments and their values. Some values in EXFOR (possibly the old ones), are far from the average and the TALYS calculation.

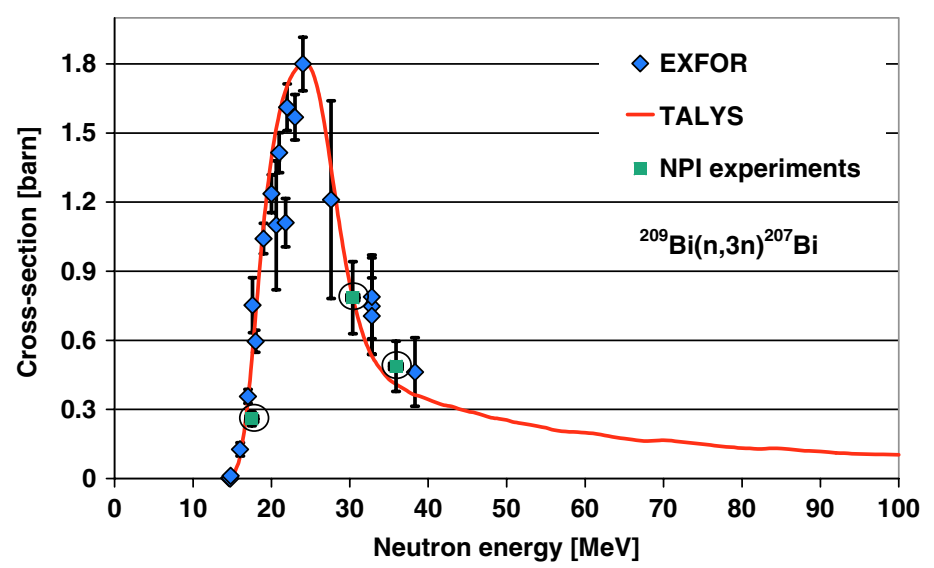

Fig. 1. Cross-section of ${ }^{209} \mathrm{Bi}(\mathrm{n}, 3 \mathrm{n}){ }^{207} \mathrm{Bi}$ reaction from EXFOR database, TALYS 1.0 calculation and NPI experiment. 
EFNUDAT Workshop “Measurements and Models of Nuclear Reactions", Paris, 2010

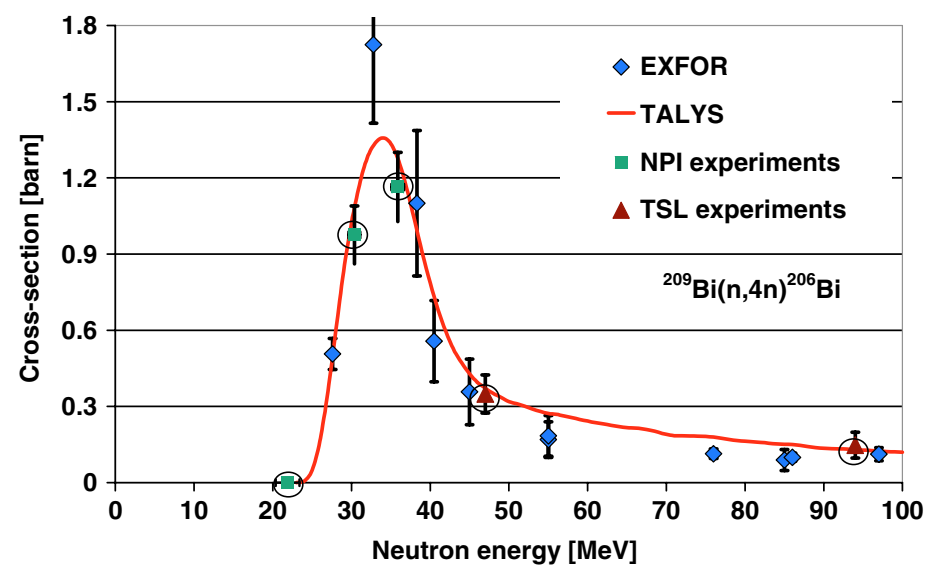

Fig. 2. Cross-section of ${ }^{209} \mathrm{Bi}(\mathrm{n}, 4 \mathrm{n}){ }^{206} \mathrm{Bi}$ reaction from EXFOR database, TALYS 1.0 calculation, TSL and NPI experiments.

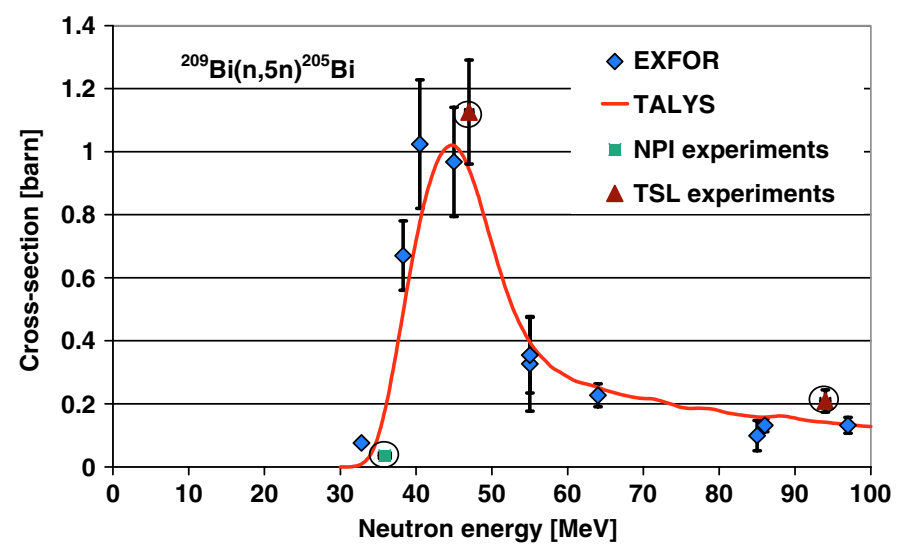

Fig. 3. Cross-section of ${ }^{209} \mathrm{Bi}(\mathrm{n}, 5 \mathrm{n}){ }^{205} \mathrm{Bi}$ reaction from EXFOR database, TALYS 1.0 calculation, TSL and NPI experiments.

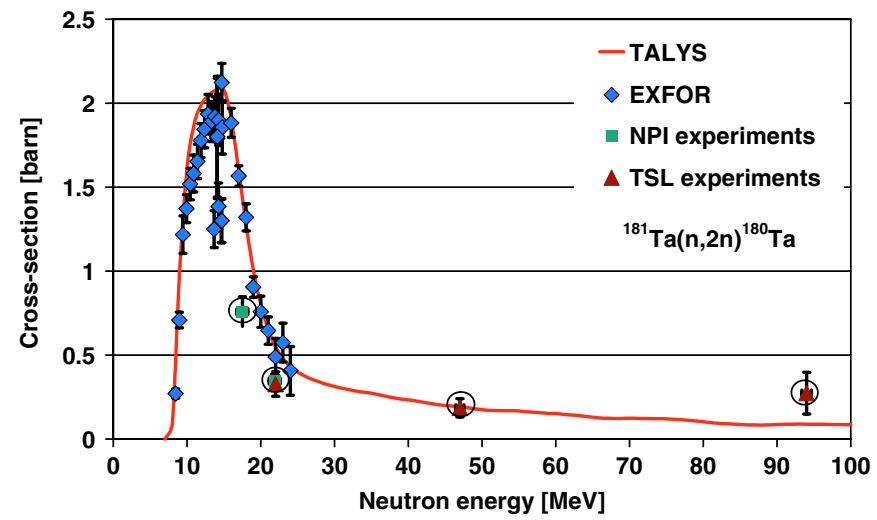

Fig. 4. Cross-section of ${ }^{209} \mathrm{Bi}(\mathrm{n}, 5 \mathrm{n}){ }^{206} \mathrm{Bi}$ reaction from EXFOR database, TALYS 1.0 calculation, TSL and NPI experiments. 
EPJ Web of Conferences

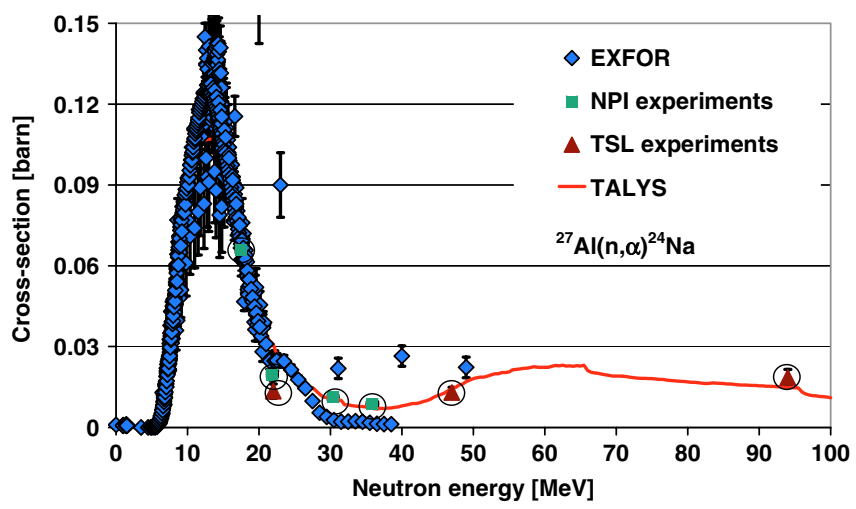

Fig. 5. Cross-section of ${ }^{27} \mathrm{Al}(\mathrm{n}, \alpha)^{24} \mathrm{Na}$ reaction from EXFOR database, TALYS 1.0 calculation, TSL and NPI experiments.

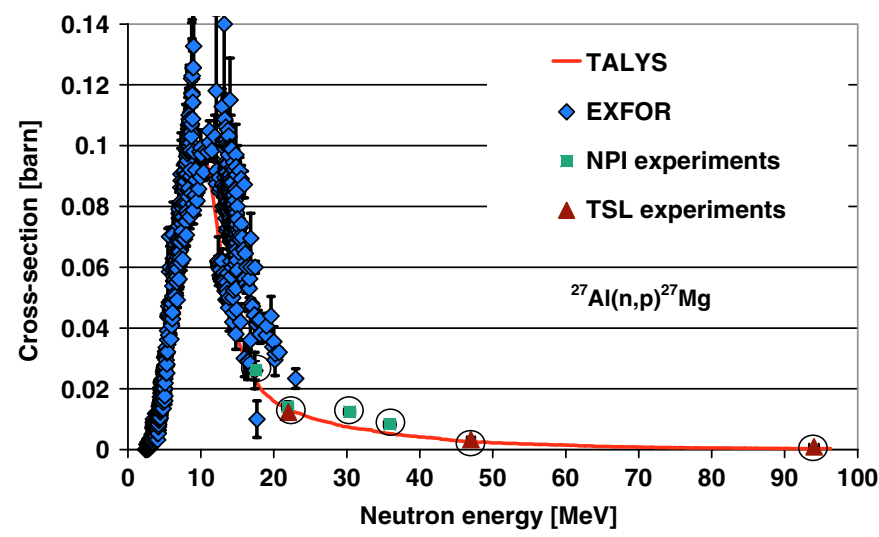

Fig. 6. Cross-section of ${ }^{27} \mathrm{Al}(\mathrm{n}, \mathrm{p}){ }^{27} \mathrm{Mg}$ reaction from EXFOR database, TALYS 1.0 calculation, TSL and NPI experiments.

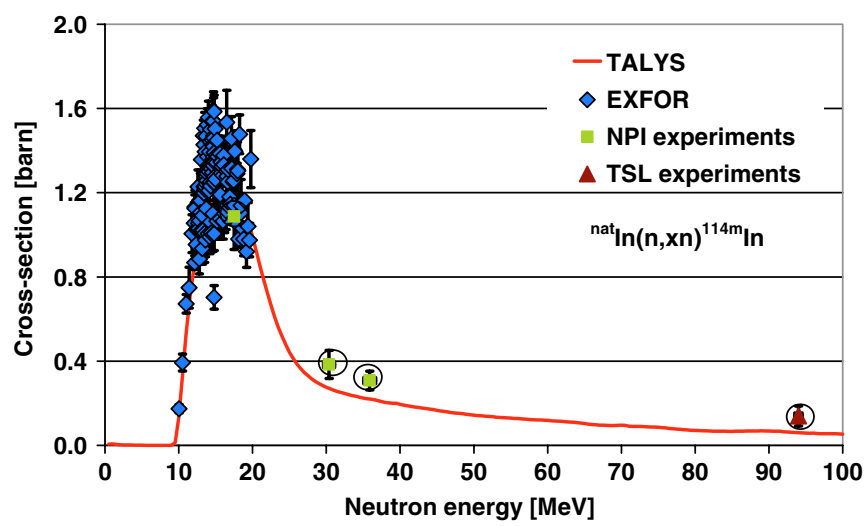

Fig. 7. Cross-section of ${ }^{n a t} \operatorname{In}(\mathrm{n}, \mathrm{xn})^{114 m} \mathrm{In}$ reaction from EXFOR database, TALYS 1.0 calculation, TSL and NPI experiments. 


\section{Uncertainty analysis}

In this type of cross-section measurements a lot of sources of uncertainties must be considered. Statistical uncertainty comes from the Gauss fit of the gamma peaks in the code DEIMOS32. It is usually between $1 \%$ and $10 \%$. Systematic uncertainties are from the neutron spectra determination (10\% for both neutron sources), beam intensity determination (5\% at NPI, respectively $10 \%$ at TSL). $1 \%$ uncertainty comes from the spectroscopic corrections; another 3\% is the uncertainty of the HPGe detector calibration. Final uncertainty was set as a square root of the second powers of particular uncertainties mentioned above.

Uncertainty of the gamma line intensities was for presented reactions not taken into account, because only strong gamma lines with negligibly small uncertainties were used.

\subsection{Background subtraction}

Approximately a half of the total neutron intensity of the beam belonged to the neutron background. The exact value depends on the energy of the proton beam. This background started a few MeV below the neutron peak and continued up to low energies, see Fig. 8. Production of observed isotopes by this neutron background could not be neglected. To subtract this background, the cross-sections of observed reactions were calculated in TALYS 1.0 [4] and a folding with the neutron spectra was performed. Ratios between the isotope production in the neutron peak and by the whole spectrum were determined. With these ratios the yields of isotopes were multiplied and the cross-sections were evaluated from these multiplied values. This procedure inserts another possible uncertainty to the cross-section data.

Different versions of TALYS (1.0 and 1.2) were examined (up to now only for gold) to see their influence on the background subtraction and thus on the final results. Differences are for most of the isotopes lower than $10 \%$.

Various settings of the level densities in TALYS 1.2 were also tested for gold isotopes; their influence on the cross-section results is shown in Fig. 9. Ld1 means constant temperature plus Fermi gas model, ld 2 is back-shifted Fermi gas model, ld3 stands for generalised superfluid model, in ld4 model are microscopic level densities taken from Goriely's table and in ld5 Hilaire's table is used for microscopic level densities. Differences between the cross-sections calculated with different models are mostly within $\pm 10 \%$. No trend was observed for any isotope or model (differences are to plus same as to minus from the basic TALYS setting ld1).

Total uncertainty of the background subtraction was up to now assessed only for gold isotopes; it is approximately $10 \%$ and has not yet been included into the total uncertainty. Further study of the TALYS influence on the background subtraction is currently under progress.

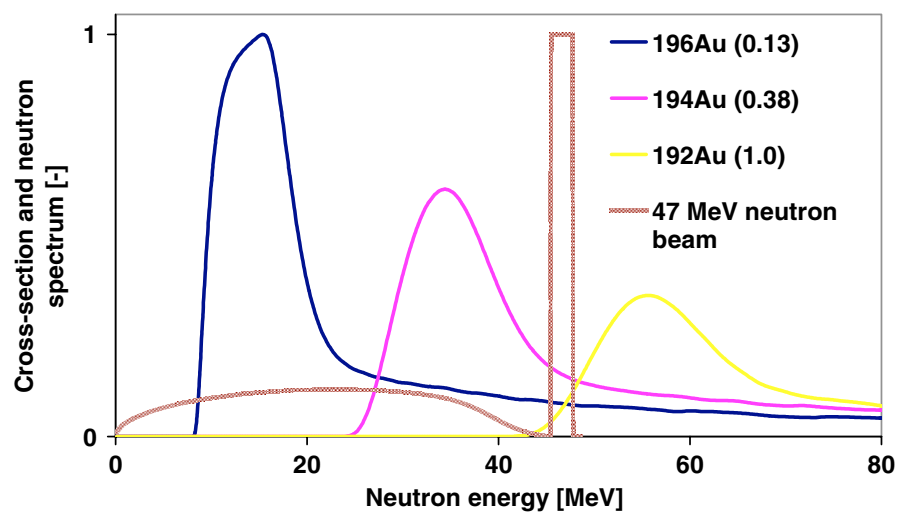

Fig. 8. TALYS 1.0 cross-sections of various $(n, x n)$ reactions in Au compared with the spectrum of $47 \mathrm{MeV}$ neutron beam at TSL (normalized values). Values in the parenthesis are ratios of peak production to total. 


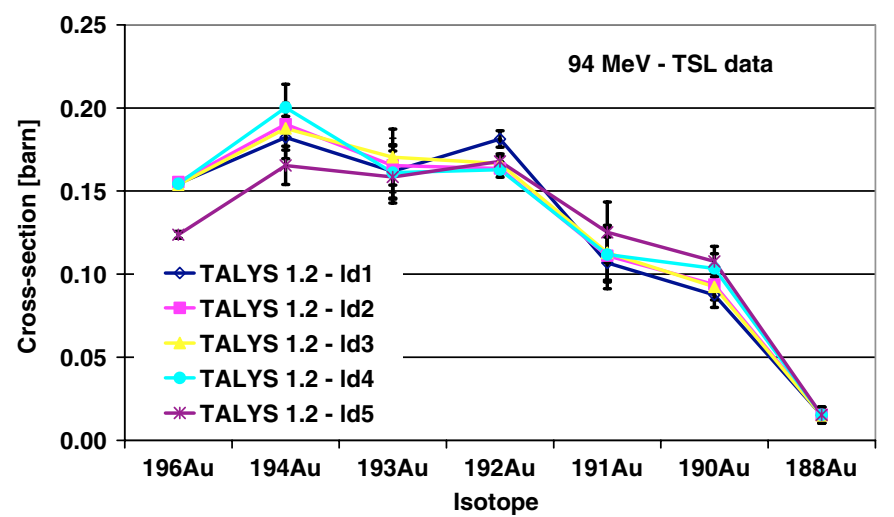

Fig. 9. Cross-sections of ${ }^{197} \mathrm{Au}(\mathrm{n}, \mathrm{xn})$ reactions calculated with different neutron background subtractions caused by various settings of level densities in TALYS 1.2.

\section{Summary}

We used quasi-monoenergetic neutron sources at TSL Uppsala and NPI Rež to measure cross-sections of $(\mathrm{n}, \mathrm{xn}),(\mathrm{n}, \alpha)$ and $(\mathrm{n}, \mathrm{p})$ reactions in $\mathrm{Al}, \mathrm{Au}, \mathrm{Bi}, \mathrm{I}, \mathrm{In}$, and Ta. We covered energy region from $17 \mathrm{MeV}$ to $94 \mathrm{MeV}$ and obtained a lot of unique data for energies, where nobody has measured so far. We got a good agreement with the data in EXFOR database. Part of our data was already published in the proceeding from of the International Conference on Nuclear Data for Science and Technology - ND2010; further results will be published soon.

We used code TALYS 1.0 to calculate observed cross-sections and subtract neutron background. Usage of new TALYS 1.2 version, same as different settings of level densities in TALYS change our results for gold isotopes within $10 \%$.

We would like to thank the staff of TSL Uppsala (especially to Alexander Prokofiev and Torbjörn Hartman) and the staff of the Cyclotron-based Fast Neutron Facility of the Nuclear Reaction Department NPI Rež (especially to M. Gotz, M. Honusek and E. Šimecková) for great support and excellent beams. Our special thanks belong to Marek Fikrle for the help with the set up of iodine samples.

This work was supported by the EFNUDAT program, grant number CTU0808214 and by the F4E program of the NRD NPI, F4E-2008-GRT-014.

\section{References}

1. A. Krása et al., Nucl. Instr. and Meth. in Phys. Res. A, 615, 70-77 (2010)

2. European Facilities for Nuclear Data Measurements, www.efnudat.eu, 19.4.2010

3. O. Svoboda et al., Procceedings of the International Conference on Nuclear Data for Science and Technology - ND2010, Jeju, South Korea (2010)

4. A. J. Koning et al., Proceedings of the International Conference on Nuclear Data for Science and Technology - ND2007, 211-214 (2007) 